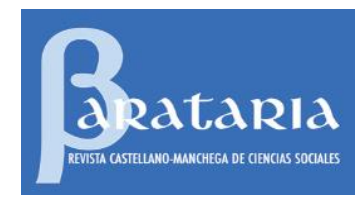

\title{
¿Es necesario regular la práctica de surf durante el verano?
}

Would it be necessary to regulate beaches with surfing zones during summer?

\author{
Diego Santos González \\ Ostelea Tourism and Management School. Madrid/España. \\ santoseko@gmail.com \\ https://orcid.org/0000-0002-2205-8962
}

\author{
Jose Miguel Moreno Carrillo \\ Universidad de Castilla la Mancha. Castilla-La Mancha/España. \\ jomimorca@gmail.com \\ https://orcid.org/0000-0001-5535-1711
}

Recibido/Received: 27/05/2020

Modificado/Modified: 02/11/2020

Aceptado/Accepted: 23/11/2020

\section{RESUMEN}

El surf evoca un estilo de vida hedonista, errante y relajado. Unos valores que encajan perfectamente con la mentalidad millennial. Desde una perspectiva turística, existen dos formas diferentes de consumir surf. Por un lado existen una serie de personas visitarán destinos principalmente por recorrer olas, es decir la presencia de olas es su motivación principal del viaje, mientras que existe otro colectivo, mucho mayor en números absolutos que quiere aprovechar su visita a la playa para practicar surf, es decir, es simplemente una actividad complementaria; una experiencia dentro del viaje. Conocer las características de ambas tipologías es realmente útil para los organismos de promoción y gestión del turismo, principalmente para anticiparse a posibles problemas que puedan surgir en sus arenales y así poder garantizar una sostenibilidad económica y social en ellos antes de que los conflictos sean demasiado graves.

\section{PALABRAS CLAVE}

Turismo de surf, regulación playas verano, zonas de surf.

\section{SUMARIO}

1.Introducción. 2. Marco teórico o revisión de literatura. 2.1 Entendiendo el surf como estilo de vida. 2.2. Surfistas y turismo. 2.3. Surf como actividad turística. 2.4. Surf para turistas. 2.5. Introducción a la regulación de las playas para la práctica de surf. 2.6. ¿Cuáles son los usos comunes? 2,7. ¿Cuáles son los usos especiales? 3.Metodología y caso de estudio. 4. Hipótesis y Resultados 5. Conclusiones. 6. Bibliografía.

\section{ABSTRACT}

Surfing evokes a hedonistic, wandering and relaxed lifestyle. Values that fit perfectly with the millennial mentality. From a tourist perspective, there are two different ways to consume surfing. On the one hand there are a number of people who will visit destinations mainly for traveling waves, that is, the presence of waves is their main motivation for the trip, while there is another group, much larger in absolute numbers that wants to take advantage of their visit to the beach to surf, that is, it is simply a complementary activity; An experience inside the trip. Knowing the characteristics of both types is really 
useful for tourism promotion and management agencies, mainly to anticipate possible problems that may arise in their areas and thus be able to guarantee economic and social sustainability in them before conflicts are too serious.

\section{KEYWORDS}

Surf tourism, summer beaches regulation, surfing zones.

\section{CONTENTS}

1.Introduction. 2. Theoretical framework or literature review. 2.1. Understanding surfing as a lifestyle. 2.2. Surfers and tourism. 2.3. Surf as a tourist activity. 2.4. Surf for tourists. 2.5. Introduction to beach regulation for surfing. 2.6. What are the common uses? 2.7. What are the special uses? 3. Methodology and case study. 4. Hypothesis and Results. 5. Conclusions. 6. References.

\section{INTRODUCCIÓN.}

El surf es un fenómeno que ha irrumpido con fuerza en España desde hace varias décadas. Regiones como Canarias o Euskadi cuentan con un gran volumen de personas cuyo centro de su vida es desplazarse sobre la superficie de las olas.

Algo que sucedió mucho antes en California y del que podemos extraer muchas conclusiones para poder entender que el surf es más que un deporte para aquellos que lo practican. Según Wayne (1976), el proceso para convertirse en surfista implicaba no sólo aptitudes sobre la tabla, sino que entre las muchas condiciones necesarias para ser aceptado por los miembros de dicha subcultura. Un factor clave para la aceptación social en la playa es saber interpretar las predicciones, es decir, haber desarrollado capacidades que ayuden a identificar las mejores condiciones de surf en diferentes playas y tener una furgoneta, ese vehículo era auténtico símbolo de status.

Sin embargo, la reciente popularización del surf en nuestro país lo ha llevado a convertirse en un producto turístico, en parte de la experiencia del viaje, sin ningún tipo de vinculación con esta subcultura ni con sus códigos sociales. Una experiencia comercial que nada tiene que ver con ese estilo de vida. Simplemente comprar una foto encima de una tabla para publicarlo en alguna red social.

El desarrollo del turismo contemporáneo, ha creado dos realidades muy diferentes en torno a los viajes de surf. Por un lado un crecimiento de surfistas viajeros que hace que cada vez haya mayor número buscando las mejores olas del planeta. Este aumento de personas buscando olas genera masificación en algunos rompientes; la respuesta al aumento de volumen creciente de turistas es generalmente hostil. Se conoce como localismo y puede llegar a producir conflictos realmente graves. Por ejemplo, en Julio de 2007, The Sydney Morning Herald informó sobre un altercado que tuvo lugar entre el bodyboarder profesional Mitch Rawlins y un grupo de varios miembros de Bra Boys (una pandilla conocida por defender lo que consideran sus playas). Al parecer, no querían dejar que Rawlins surfear allí por lo que estalló una discusión que terminó en agresión cuando Rawlins recibió un puñetazo en la cabeza. Un portavoz de los Bra Boys confirmó que había habido "algún tipo de incidente pequeño", pero negó cualquier violencia importante.

Por otro, el crecimiento de las escuelas de surf con la intención de ofrecer experiencias sobre olas. Como los turistas son un mercado importante, tienen un volumen alto de alumnos durante el verano, pese a coincidir con uno de los momentos del año con menor presencia de olas, lo que se traduce en la necesidad de aprovechar la mínima espuma para las clases, lo que en muchas ocasiones se da en la orilla, pudiendo suponer un conflicto entre surfistas y bañistas. 
Es importante satisfacer las necesidades de las nuevas tendencias turísticas. Pero debemos tener en cuenta que destinos como Sanxenxo y O Grove son destinos maduros en el nicho de sol y playa que no deben generar insatisfacción en aquellos turistas fidelizados con una cuestión de tan fácil solución como la del uso del espacio destinado al baño.

Es cierto que la industria turística necesita que las personas consuman el mayor número de productos en los diferentes destinos. En su momento estos destinos, especialmente Sanxenxo, estaban muy vinculados con el consumo de ocio nocturno el cual sufrió numerosas reformas y regulaciones para causar el menor impacto posible sobre aquellos turistas que preferían descansar.

Las diferentes regulaciones intentan generar una sostenibilidad en el turismo para que el ciclo del producto turístico no entre en declive con excesiva rapidez. Desde un punto de vista normativo, los diferentes destinos pueden ser más o menos flexibles, muchas veces con el objetivo de no frenar el desarrollo económico. Como reproduce Santos (2016) en su tesis doctoral, haciéndose eco de Vidal Díaz: "El ocio y el turismo, además de contribuir a proporcionar felicidad y placer al individuo -y para ello han de despertar emociones-, también contribuyen a servir de herramienta para el sistema capitalista basado en el consumo."

Con la evolución y desarrollo del turismo, los destinos de sol y playa se han adaptado a las necesidades de los turistas, especialmente tratando de satisfacer las necesidades de diferentes públicos con el objetivo de resultar lo más atractivos posibles.

Ante esta realidad, uno de los productos turísticos que mejor encaja es el de la práctica de surf. Circunstancia que ha llevado a algunos destinos a optar por regular y planificar el turismo de surf. Lamentablemente en estudios precedentes (Santos, 2016) se vio que no siempre se está realizando una gestión o planificación de turismo de surf de manera eficiente, por lo que en este artículo compararemos algunos casos que ejemplifiquen la disparidad de soluciones tomadas en diferentes arenales, centrándonos en el estudio del caso de la playa de A Lanzada, uno de los principales destinos de turismo estival de surf en Galicia.

\section{MARCO TEÓRICO O REVISIÓN DE LITERATURA.}

Nos encontramos ante un objeto de estudio que no ha sido muy estudiado ni documentado; quizás esta sea una de las razones por las que las administraciones han reaccionado tarde para tratar de gestionar el crecimiento de la práctica de surf. El desconocimiento sobre los surfistas, sobre las condiciones ambientales para su práctica y la necesidad para solucionar un problema en crecimiento, unido a la complejidad legal del uso y explotación del dominio marítimo terrestre lleva a que la gestión y la planificación de la práctica de surf sea un caos en muchos destinos turísticos, pudiendo afectar a otros agentes turísticos que comparten espacio.

Antes de reflexionar sobre la importancia económica y social que puede tener para un destino una correcta planificación en materia de turismo de surf, se considera necesaria una revisión conceptual que nos ayude a tener claro de qué estamos hablando, ya que bajo el paraguas del concepto turismo de surf, existen realidades muy diferentes. Como avanzábamos en la introducción, no es lo mismo que un surfista se desplace a buscar las mejores olas que una persona quiera practicar surf durante sus vacaciones.

\subsection{ENTENDIENDO EL SURF COMO ESTILO DE VIDA.}

El surf contemporáneo no es sólo un deporte, sino que es una realidad industrial, económica y social muy compleja, la cuál que se ha desarrollado a partir de una práctica milenaria, a priori 
tan sencilla, que consistía simplemente en desplazarse sobre la superficie de las olas. El surf y su evolución han sido estudiados por disciplinas tales como la historia (Esparza, 2014; Kampion, 2003), el periodismo (Beattie, 2001; Henderson, 1999, 2001; Ormrod, 2005a, 2005b; Rutsky, 1999; Stedman, 1997) o las ciencias del deporte (Booth 1995; Nazer 2004; Stranger 1999); la gran mayoría investigando la práctica del surf en aspectos puramente deportivos.

Tal y como se afirma en Santos (2017), a día de hoy existen miles de individuos que comparten el estilo de vida que desprende el surf. Para poder comprender estas sensaciones y estos sentimientos, no basta con un acercamiento superficial a sus practicantes. Será necesario adentrarse en lo más profundo de esta realidad para poder conocerlos y describirlos. Comprender que no todos los surfistas son iguales, aunque la gran mayoría estén en la búsqueda constante de una ola perfecta que quizás llegue pronto o que quizás no exista. Para muchos, el surf se convierte en el eje central de sus vidas, girando el resto de actividades en torno a temporada de olas, vientos y mares. Las ansias de muchos individuos por surfear son tan grandes que no puede esperar a que la naturaleza envíe olas a nuestras costas y, como si de una conquista de tratase, pretende incluso producirlas artificialmente.

Wayne (1976) profundizó en el estudio del estilo de vida de los surfistas analizando el surf como subcultura, proponiendo una diferenciación de los surfistas según su grado de implicación en la práctica. Para poder conocer dicha implicación elaboró el índice gracias al cual pudo concluir que efectivamente no todos los surfistas tienen el mismo grado de implicación.

La metodología de Wayne para elaborar este índice no ha sido sólo a través de la observación y experiencia, sino que a partir de las respuestas obtenidas de un cuestionario que distribuyó en la prestigiosa Surfer Magazine. Diseñó un instrumento para medir la implicación de los surfistas en el surf, bautizado como Surfing Index Involvement (SII), cuyo proceso de elaboración es argumentado paso a paso en su tesis doctoral (1976) y se calcula con la suma del número de años surfeando (multiplicado por diez), de la frecuencia surfeando y de la proporción de amigos (multiplicado por veinticinco). Una vez obtenido este índice y de haber diferenciado entre poca implicación, implicación moderada y alta implicación, posteriormente estableció diferentes correlaciones con este índice para tratar de comprender qué factores podían tener más influencia, como el género, la edad, los estudios, la condición social o la distancia a la playa, entre otros. Demostrando que en su estudio existía una fuerte correlación entre el grado de implicación con el surf y sus estudios. "los surfistas que están más implicados en la práctica del surf eligen carreras que minimicen su interferencia sus actividades vinculadas con el surf" (Wayne, 1976; p22), que prácticamente no había surfistas "no blancos" o cómo aunque su hipótesis era que las clases sociales más altas estaban más implicados en la práctica del surf, nunca pudo demostrarlo. Si pudo demostrar que aquellos más implicados "tienden a mudarse a vivir a zonas con buenas olas si no viven ya en ellas" (p30).

Considera Álvarez Sousa (2011) que el lenguaje de los símbolos es el que debe dominar el innovador turístico, con el objetivo de que todo tipo de recursos puedan impregnarse de significado y poder ofrecérselo a determinados tipos de turistas. "La creatividad puede llegar a tal nivel que, a veces, se pueden crear necesidades específicas y estilos de vida en los turistas a partir de los objetos, servicios y productos turísticos que le ofrecemos" (Álvarez Sousa, 2011:142). Además, este mismo autor considera que, en ocasiones, el turismo ya no es sólo un complemento del mundo del trabajo, sino que se convierte en el objetivo de muchas personas y el trabajo es el complemento que lo permite. Atendemos por tanto a una inversión de los factores del estilo de vida de algunas personas. Si bien también considera que este innovador, 
que crea objetos, servicios y productos turísticos no puede ser una persona aislada, sino que tiene que ser un equipo de expertos.

Los organismos institucionales de promoción y gestión de las playas, por no tener claro qué o quién es realmente un surfista cuyo estilo de vida gira entorno a la búsqueda de olas, desarrollan iniciativas que no los satisfacen desde el punto de vista turístico, y lo que es peor, en ocasiones realizan acciones completamente perjudiciales como restringir servicios de ducha durante el invierno, limitar el uso del aparcamiento o limitar las zonas de práctica de surf.

Surcar las olas con la tabla de surf en Barcelona es tarea harto complicada. Y el natural estado calmo del Mediterráneo no tiene la culpa. En verano, por respeto a los bañistas y en inverno, por la ordenanza de Convivencia, que establece sanciones de entre 750 y 1.500 euros cuando no se respeten "las señalizaciones sobre las condiciones y lugares de baño" o haya "bandera roja"

Tal y como el Ajuntament reconoce, las playas de Barcelona son usadas durante todo el año, siendo la primavera y el verano las estaciones de mayor demanda. Por este motivo, hay actividades que no se pueden realizar en esos meses, como pasear perros, gatos y otros animales de compañía. La limitación se extiende entre Semana Santa y septiembre.

Los surfistas no están de acuerdo porque, según explican, "somos personas centradas". Y recuerdan que "nadie mejor que nosotros sabe dónde va a romper una ola o si estamos capacitados para subirnos a ella", asegura Chiodi. 20 Minutos (06/02/2012)

\subsection{SURFISTAS Y TURISMO}

El turismo de surf se refiere a aquellas personas cuya motivación principal del viaje es desplazarse sobre la superficie de las olas. Comenzó probablemente con surfistas precoloniales que llevaban a cabo viajes de exploración en busca de nuevos spots de surf hace más de mil años en las islas de Hawai (George, 2000). A día de hoy se ha convertido en un subsector altamente industrializado de la industria turística que cuenta con más de ciento cincuenta agencias de viajes especializadas en este nicho en todo el mundo (Borden, 2005; Warshaw, 2004) y miles de operadores menores especializados en fletar barcos de lujo a través de los océanos Índico y Pacífico. Este tipo de turismo abastece desde turistas que buscan hoteles de lujo con acceso exclusivo a olas de clase mundial como sucede en Fiji y en Maldivas, a perfiles más modestos que buscan alojamiento barato en construcciones modestas o incluso en casas de familias anfitrionas a lo largo de los destinos del mundo surf. (Ponting, 2008:2).

Esta industria es geográficamente muy diversa e incluye los más famosos rompientes en Europa, África, Asia, Australasia, el Pacífico y las Américas. Las estimaciones más optimistas sugieren que la industria internacional del turismo de surf atiende a más de cien mil turistas anualmente y la facturación anual se estima en este ámbito en cifras cercanas a cientos de millones de dólares (Ponting, 2008). Por tanto, es evidente que se ha convertido en un importante segmento de los ingresos de la industria turística en algunos países en vías de desarrollo pues, por ejemplo, supuso el dos y el diez por ciento de las llegadas de turistas a Fiji (Tebbut, 2006) y Papúa Nueva Guinea (Ponting, 2008) en 2005, respectivamente. A pesar de lazos históricos popularmente percibidos entre el surf moderno y una divergencia de éste con los principales imperativos capitalistas, la industria del surf, incluyendo la industria del turismo de surf, perpetúa la lógica de acumulación de capital con poco respeto por las externalidades que produce como son los impactos ambientales y socioculturales (Barilotti, 2002; Ponting, 2001).

El surf se ha convertido sin apologías en un componente más del capitalismo global 
hegemónico (Buckley, 2003; Hammerschmidt, 2004). El cada vez más creciente número de consumidores compra el ideal representado por la ficción, el escape soñado de un Nirvana del surf (Henderson, 1999, 2001; Lanagan, 2002; Ormrod, 2005a, 2005b; Reed, 1999; Scheibel, 1995; Stedman, 1997), mientras que un número también cada vez mayor de comunidades remotas en algunas de las regiones menos desarrolladas del planeta se convierten en anfitriones pasivos de este número creciente de turistas de surf a la vez que son marginados de los beneficios económicos que el turismo de surf puede proporcionar.

A mediados de los noventa los nuevos destinos estaban tan masificados como las playas más populares de California o Australia, con ocasionales peleas por las olas, por lo que los viajeros empezaron a preguntarse si realmente tenía sentido viajar a estas latitudes.

En ocasiones parece que los surfistas olvidan el factor fundamental de viajar, que es experimentar con otras culturas ya que en el barco surfean con otro grupo de occidentales, beben cerveza y juegan a las cartas. Se retoma entonces la vieja distinción entre viaje y turismo. El viajero acepta dificultades durante su búsqueda de la auténtica experiencia, mientras que el turista es el producto de la industria del ocio. (Ponting, 2008).

Para la mayoría de lugares que desean atraer surfistas, una de las estrategias más utilizadas por los organismos de promoción que quieren atraer a surfistas a sus destinos es la celebración de campeonatos. Los ejemplos más relevantes en nuestra zona de estudio fue la organización de dos ediciones del Rías Baixas Pro, campeonato que contaba con los mismos promotores que el Pantín Classic.

Sin embargo, este tipo de estrategias, que por su repercusión mediática aumentan rápidamente la percepción de práctica de surf en la zona. En la comunidad surfera tradicionalmente se ha asociado al lucro y en numerosas ocasiones eran recibidos con hostilidad.

Ya en la década de los 70, los surfistas hawaianos se vieron compitiendo por las olas con hordas de australianos, californianos y otros visitantes, además de encontrarse en muchas ocasiones con la entrada restringida a la playa durante los mejores días del invierno por la celebración de campeonatos. Estos campeonatos no solo producían estas restricciones de acceso, sino que además atraían a muchos surfistas que aspiraban a ser profesionales y surfearían en las playas cercanas cuando no fuese su turno de competición. Un caldo de cultivo para el surgimiento de bandas de surfistas que se organizaban para defender sus rompientes de la presencia de foráneos, como fue el caso de los "Black Trunks".

\subsection{SURF COMO ACTIVIDAD TURÍSTICA.}

El atractivo del deslizamiento sobre la superficie de las olas ha sido realzado hábilmente por los responsables de marketing de empresas de todo el mundo (Henderson, 1999, 2001; Lanagan, 2002; Ormrod, 2005a, 2005b; Reed, 1999; Scheibel, 1995; Stedman, 1997), en gran medida porque responde a muchas de las necesidades de consumo de la sociedad contemporánea. El surf ha pasado de no tener casi protagonismo a convertirse en el medio trasmisor de la imagen de muchas compañías. Además, algunas marcas no se han limitado a realizar un único anuncio con estética surf, sino que van mucho más allá y es el tipo de contenido al que quieren vincular su marca, lo que en marketing se conoce como branded content.

Por estas razones, en un mundo cada vez más hedonista, en el que las personas quieren demostrar una vida cada vez más rica en experiencias, las personas utilizan para su vida personal estrategias de marketing similares a las de las grandes empresas. Por lo que podemos 
afirmar que el surf ha resultado ser una práctica que se ha amoldado mejor que casi ninguna otra a los valores más contemporáneos de la sociedad.

Si bien existen artículos periodísticos o de opinión que pretenden establecer tipologías de surfistas, muy pocas son las clasificaciones de surfistas que tienen base científica. De entre ellas destaca la clasificación que diferencia a los surfistas atendiendo a su técnica y a su capacidad para realizar maniobras en determinadas olas, que encontramos en Mead (2003) desarrollada por Butt (2001).

Consideramos que es un grandísimo trabajo pues pese a tratarse de un análisis subjetivo, se ha tratado de estandarizar a los surfistas valorando las capacidades de ejecución de maniobras de cada uno de ellos en diferentes olas, otorgando así un valor a su capacidad técnica entre 1 y 10 .

Tabla 1.Ratio técnica surfistas y olas.

\begin{tabular}{|c|c|c|c|}
\hline \multicolumn{4}{|c|}{$\begin{array}{l}\text { Ratie de técnica de surfiatas. Los ratios son independientes de la calidad } \\
\text { del romplente y de la dificultad de la ola. }\end{array}$} \\
\hline RATO & Descrpolin & $\begin{array}{l}\text { Uinte del angio } \\
\text { gentes }\end{array}$ & $\begin{array}{l}\text { Tarato de } \\
\text { tads }\end{array}$ \\
\hline 1 & 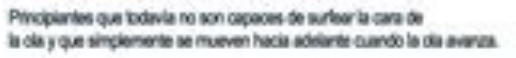 & 90 & 0.70100 \\
\hline 2 & 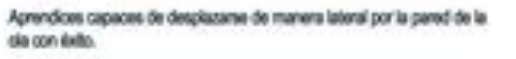 & $\pi$ & 0.65150 \\
\hline 3 & 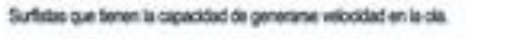 & 60 & 0.502 .50 \\
\hline 4 & 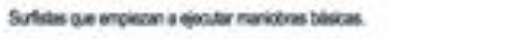 & 56 & 0.550400 \\
\hline 5 & 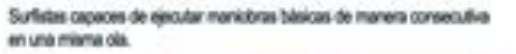 & 50 & 0.500400 \\
\hline 5 & 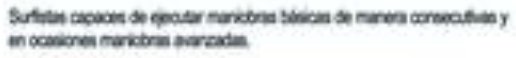 & 40 & $0.45 \times 4,00$ \\
\hline 7 & 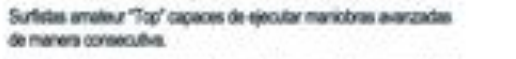 & 22 & 0.450400 \\
\hline 8 & 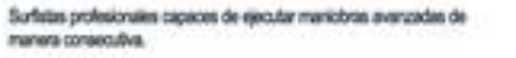 & 27 & $0.25 \times 4,00$ \\
\hline 9 & 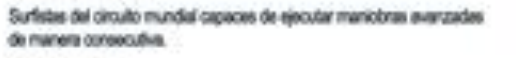 & No vicarado & 0.300400 \\
\hline 10 & Sulnestites & $\mathrm{Nb}$ alcanzado & $03 \times 400$ \\
\hline
\end{tabular}

Fuente: Santos 2015.

Según lo observado en la figura anterior podemos ver que se ofrecen valores entre 1 y 4 para diferentes tipologías de principiantes, valores entre 5 y 6 para surfers capaces de realizar maniobras básicas, 7 serán aquellos surfistas amateurs que realizan maniobras avanzadas complejas, 8 surfistas profesionales capaces de realizar maniobras avanzadas complejas, 9 surfistas profesionales del primer nivel (antiguo Top 44) y 10 surfistas del futuro.

Aunque se podría reflexionar sobre la idoneidad de dicho ranking en función de la técnica, esta clasificación nos revela que podemos hablar a nivel general de surfistas principiantes, surfistas de un nivel intermedio y surfistas de nivel avanzado. Mención especial merecen aquellos que dentro del nivel avanzado son surfistas profesionales.

Vemos también en la tabla dos parámetros que varían según el nivel del surfista. Primero vemos el del límite del ángulo de rotura, que hace referencia, en número de grados al ángulo 
que establece la concavidad de la ola y en segundo lugar el tamaño mínimo y máximo de la misma, expresado en metros.

Sin duda, no todos los surfistas tienen la capacidad técnica de surfear las mismas olas.

\subsection{SURF PARA TURISTAS}

Álvarez Sousa (2011) considera que, en las acciones turísticas, el constructivismo sólo se da cuando existe una predisposición emocional para ver "una determinada actividad desde la óptica emocional del turista que le va a llevar a un determinado disfrute" (Álvarez Sousa, 2011:150); de no ser así, podría parecer como una práctica absurda carente de la capacidad de producir deseo. En este sentido se concluye que para crear un recurso turístico se ha de crear una situación emocional adecuada que en algunos productos turísticos necesita incluso de una preparación física y mental previa.

Aunque tradicionalmente el surf había sido una práctica autodidacta, en este contexto turístico se antojan necesarias empresas que ayuden a aquellos que lo deseen a iniciarse en la práctica del surf, bien a través del alquiler de material, o fundamentalmente ofreciendo cursos de iniciación.

En la tesis Doctoral "El mundo sobre las olas. Perspectivas de desarrollo turístico a través del surfing" (Santos, 2015), se distribuyó un cuestionario para conocer el comportamiento de los surfistas, en el que se evidenció que casi el $65 \%$ de los encuestados había realizado al menos un curso de iniciación al surf. De ellos el $43 \%$ tuvieron una duración de entre dos y cinco días, mientras que casi un $12 \%$ duraron entre seis y catorce días. Santos asegura que muchos de los cursos que se ofrecen como campamentos de surf son de siete días y seis noches, por ello ha establecido esta temporalidad.

El autor continúa analizando a los surfistas de nivel medio y avanzado, observando un importante descenso de surfistas que ha realizado un curso de perfeccionamiento.

Parece evidente que la industria turística local intenta aprovechar el surf como recurso turístico, siendo estos en su gran mayoría principiantes, por lo que en muchos arenales podrían utilizar la misma zona de playa que los bañistas.

\subsection{INTRODUCCIÓN A LA REGULACIÓN DE LAS PLAYAS PARA LA PRÁCTICA DE SURF}

Este estudio, trata de ofrecer un acercamiento a la percepción de los usuarios, para una comprensión de la realidad sobre la gestión de las playas desde un punto de vista jurídico, recomendamos la revisión de "La ordenación y gestión de las playas" (Lobo, 2016) quién realiza un magnífico acercamiento a las singularidades que en esta materia posee nuestro país.

Consideramos, más allá de la vaga normativa actual, que por la complejidad de la ordenación del territorio en España deja en manos de diferentes organismos su eventual aplicación, se hace necesaria, como existe en otros países (véase la Ley 27280 conocida como Ley de rompientes en el Perú).

Un marco normativo que dé respuesta a las necesidades sociales que están surgiendo en tantas playas de nuestro país. Unas normas que deben garantizar la sostenibilidad social, ambiental, cultural y turística de los rompientes en los que se practica surf. Ofreciendo una respuesta que pueda satisfacer los intereses tanto de surfistas, empresas prestadoras de servicios de surf y el resto de usuarios que disfrutan de las playas.

Según Santos (2015) el aumento de surfistas en Sanxenxo se hace evidente especialmente durante la última década, con el boom que sufrió el surf. Esta realidad evidenciada por el 
investigador es contrastada al preguntarle a los residentes y turistas habituales de la zona:

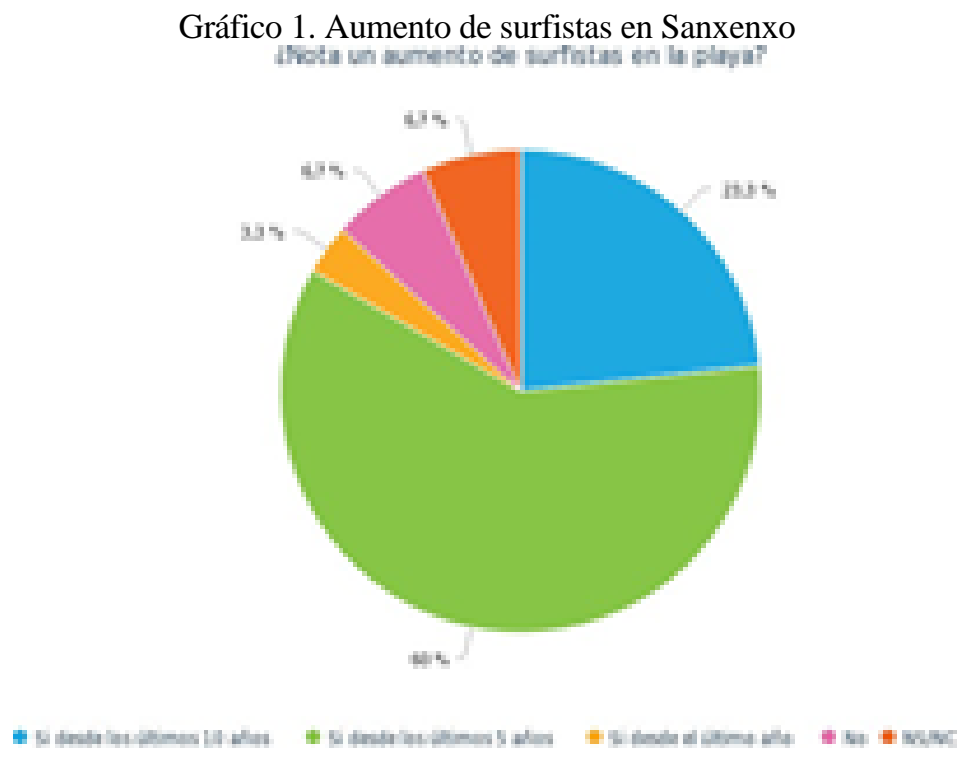

Fuente: Santos 2015.

La percepción de la población evidencia el comienzo de un aumento de surfistas desde hace aproximadamente quince años y se hace mucho más plausible en los últimos diez. Fruto de este boom es el surgimiento de numerosas escuelas para introducir a quien lo desee en esta práctica. Si bien la convivencia entre ellas y entre los demás surfistas es cordial como norma general durante el año, la temporada estival ha provocado numerosos conflictos de intereses entre las escuelas, los surfistas y los bañistas. Conflictos que han llevado a que el ayuntamiento se haya visto en la necesidad de adoptar medidas, limitando zonas de surf dónde los bañistas no pueden estar en el agua y licitando espacios para que las escuelas de surf exploten la playa.

Sin embargo, los usuarios que disfrutan de las olas de manera habitual, se muestran poco conformes con la limitación de las zonas de surf, con un $70 \%$ en desacuerdo de la regulación propuesta y un $30 \%$ no sabe o no contesta, con un $0 \%$ de los surfistas encuestados que la considere adecuada en el año 2015.

\section{Imagen 3. Delimitación de zonas de surf en Sanxenxo (surfistas)}

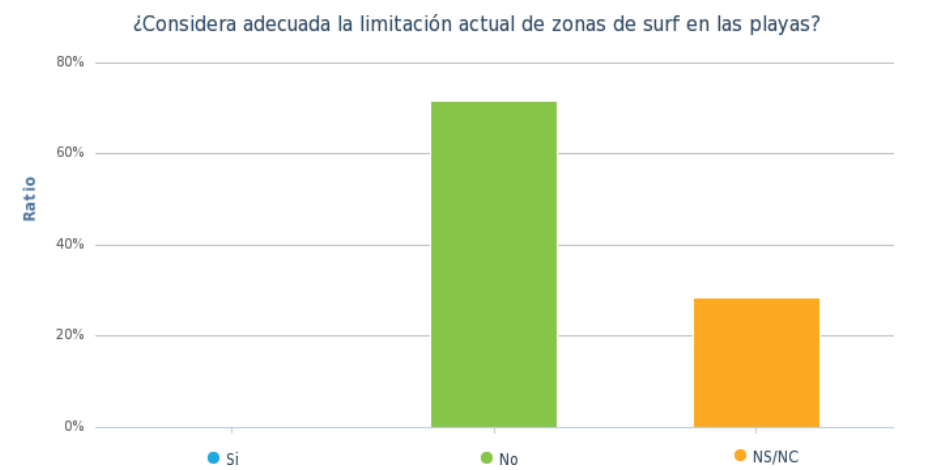


Además un $60 \%$ de los surfistas encuestados consideran que se debe limitar el volumen de escuelas durante todo el año.

Imagen 4. Limitación de número de escuelas (surfistas)

¿Cree que debería limitarse el número de escuelas en las playas?

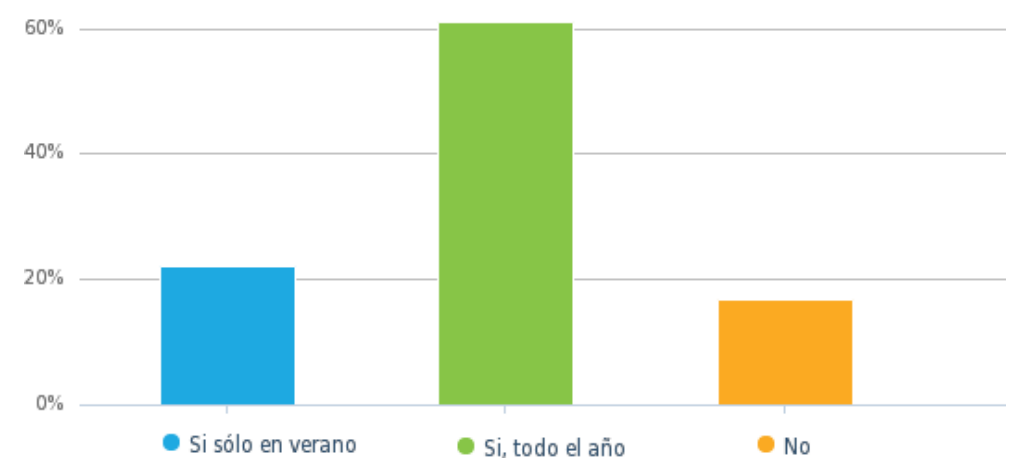

En el caso de Sanxenxo Santos asegura que con objeto de desestacionalizar la demanda, una necesidad que se ha observado, en invierno podría atraer surfistas regionales de nivel medioalto, mientras que en verano tiene que centrarse en aquellos turistas que ven el surf como producto turístico, pero se antoja conveniente una estrategia que trate de convertirlos en surfistas con un mayor nivel de implicación cultural, con el objeto de buscar retorno en temporada no estival como sucede en destinos de Cantabria y Asturias.

Los prestadores de cursos de surf, son normalmente de naturaleza muy diversa, lo que supone una diferente relación con Hacienda en cuanto a impuestos y sobre la responsabilidad civil ante un eventual accidente. Fundamentalmente estas entidades son clubs deportivos y sociedades limitadas. Sobre este tema reflexionó José María de Paz en un artículo de la revista Cerveza Salada, que pese a que el original no está ya disponible en su web, puede consultarse en el blog Mareas vivas y en el que el jurista pontevedrés asegura que "la mayoría de las escuelas de surf son un negocio, cuando digo que son un negocio significa que sus titulares tienen ánimo de lucro, y por tanto el explotarlas bajo la figura jurídica de «asociación» (club) se está incurriendo en un fraude de ley como una catedral..." finaliza con la afirmación de que toda "escuela con ánimo de lucro debe ser explotada por una persona física o jurídica", explicando además las diferentes posibilidades para estar al tanto de los pagos a Hacienda y Seguridad Social.

Además de cierto laxismo en la forma jurídica de los prestadores de servicios, estas durante años explotaban los rompientes a su libre albedrío.

En el año 2016 la Demarcación de Costas de Galicia publicaba mediante un anuncio, la información al respecto de las instalaciones de temporada en las playas de la provincia de A Coruña, entre las que se hacía referencia habría que tener autorizaciones para realizar actividades en la playa incluyéndose a las escuelas de surf en todas sus modalidades.

El texto recoge una serie de características que deben cumplir las Empresas y Clubes que quieran dar clases de surf para poder conseguir los permisos. 
La situación creó incertidumbre entre las escuelas pues algunos Ayuntamientos de diferentes puntos de la geografía gallega habían establecido concesiones para la explotación de la actividad de surf en diferentes arenales.

Algunas escuelas trataron de tensar la situación afirmando que todo respondía a afán recaudatorio por parte de la Administración. Especialmente crítico se muestra el dueño de la escuela de surf Namarea tanto en redes sociales como en conversaciones informales mantenidas con él.

Lo cierto es que pare evidente que cuando existe lucro, debe haber alguna regulación que obligue a pagar impuestos, especialmente cuando este proviene de la utilización de un espacio público.

Muchos de los responsables de escuelas argumentaban "Yo pago mis impuestos", el problema surge en que la Constitución de manera general y Ley de Protección y uso sostenible del litoral y de modificación de la Ley de Costas y Reglamento General de Costas, de manera más concreta hablan de que existen unos bienes públicos y entre ellos se encuentran los bienes delimitados por el dominio público marítimo terrestre (DPMT) el cual incluye a las playas.

La normativa de Costas establece, entre muchas otras cosas, de dos tipos de usos para este DPMT (o terreno público): los usos comunes, que no necesitan autorización, y los usos especiales que si la necesitan. Además, la utilización del Dominio Público a través de sus usos comunes debe realizarse de manera pública y gratuita.

\section{6 ¿CUÁLES SON LOS USOS COMUNES?}

El Real Decreto 876/2014, de 10 de octubre, por el que se aprueba el Reglamento General de Costas. establece que: "La utilización del dominio público marítimo terrestre y, en todo caso, del mar y su ribera será libre, pública y gratuita para los usos comunes y acordes con la naturaleza de aquél, tales como pasear, estar, bañarse, navegar, embarcar y desembarcar, varar, pescar, coger plantas y mariscos y otros actos semejantes que no requieran obras e instalaciones de ningún tipo y que se realicen de acuerdo con las leyes y reglamentos o normas aprobadas conforme a la Ley de Costas. En definitiva, los usos generales del DPMT corresponden por igual a todos los ciudadanos indistintamente, de modo que el uso de unos no impida el de los demás ciudadanos."

\section{7 ¿CUÁLES SON LOS USOS ESPECIALES?}

Tal y como Establece el Real Decreto 876/2014, de 10 de octubre, por el que se aprueba el Reglamento General de Costas sobre usos especiales: "Son los que requieran la ejecución de obras e instalaciones (por ejemplo, un puesto de salvamento) o los que, sin requerir obras, tengan especiales circunstancias de intensidad, peligrosidad o rentabilidad. Necesitarán la existencia de un título administrativo, que puede ser el de autorización o concesión.

Estarán sujetas a previa autorización administrativa las actividades en las que, aun sin requerir obras o instalaciones de ningún tipo, concurran circunstancias especiales de intensidad, peligrosidad o rentabilidad, y asimismo la ocupación del dominio público marítimo terrestre con instalaciones desmontables o con bienes muebles. (...) El plazo de vencimiento será el que se determine en el título correspondiente, y no podrá exceder de 4 años.

Por otra parte, toda ocupación de los bienes de dominio público marítimo terrestre estatal con obras o instalaciones no desmontables o con instalaciones desmontables, por plazo superior a 4 años, estará sujeta a previa concesión otorgada por la Administración del Estado (...). Las concesiones se otorgarán sin perjuicio de tercero y dejando a salvo los derechos 
preexistentes. El plazo será el que se determine en el título correspondiente y no podrá exceder de 75 años."

En virtud de lo expuesto creo que nadie puede negar que las escuelas de surf, tanto con instalación (desmontable o fija) como sin ella se encuentran el apartado de usos especiales del dominio público marítimo terrestre.

Tras este repaso teórico sobre la realidad social y legal, propondremos un acercamiento empírico con el que tratar de hacer un análisis de percepción sobre la regulación de las escuelas a día de hoy, cuando ha pasado el tema lo suficiente para dejar de ser conflictivo por su novedad y poder extraer conclusiones que nos permitan evaluar esta regulación y ofrecer opciones de mejora.

\section{METODOLOGÍA Y CASO DE ESTUDIO.}

Analizar el turismo implica conocer diferentes perspectivas teóricas para observar la realidad (Álvarez Sousa, 2011:14) y para ello se propondrán las visiones micro, macro y meso. Además de estas visiones, el análisis se efectúa desde los aspectos objetivos y subjetivos y, las perspectivas de consenso y de conflicto.

Por tanto, en el seno de esta investigación nos encuadramos en una perspectiva multiparadigmática e integrada bajo el modelo de sistema abierto, multiparadigmática siguiendo la concepción de Fiedrischs que considera que en un mismo momento se está trabajando en más de un paradigma. Integrada pues, pese a la existencia de teóricos que consideran que sólo debe primar una perspectiva, para nuestra investigación se ha considerado que tanto las visiones, los análisis y perspectivas citadas anteriormente no se deben tomar como realidades contrarias o enfrentadas sino que son complementarias. Se considera también que nos encontramos en un paradigma de sistema abierto ya que tanto el surf como el turismo se enmarcan dentro de una dinámica de interconexiones y procesos de retroalimentación en relación con los distintos subsistemas de la sociedad.

Algunos autores como Ritzer (1993) proponen elaborar paradigmas en torno a ejes del continuum (Liedloff y Martin, 2009) macroscópico-microscópico y continuum objetivosubjetivo. Además de la tradicional polaridad micro-macro, en esta investigación se tratará de analizar la percepción, un aspecto de la realidad realmente subjetiva.

A los continuos micro-macro y objetivo-subjetivo, presentados por Ritzer (1993), Álvarez Sousa (2011), añade el factor consenso-conflicto, ya que el autor considera que según la postura que tome el investigador veremos la sociedad como algo a conservar o a transformar. Antes de aventurar ninguna posición, Álvarez Sousa (2011:16) considera que "todo extremo es malo y cree que lo ideal es realizar un análisis centrado en qué pueden aportar ambas posturas y así tomar decisiones a la hora de concretar políticas de turismo".

Desde un punto de vista puramente metodológico, se ha optado por aplicar en esta investigación una estrategia basada en la teoría fundamentada o "grounded theory" por encajar perfectamente en muchos aspectos generales de nuestra investigación, pues su aplicación se da principalmente en torno a ámbitos muy delimitados o particulares de la realidad social o cultural sobre los cuales la teoría formal no ha desarrollado una comprensión ni una conceptualización específica.

Corbin y Strauss (2002) consideran que esta teoría derivada lleva a que se generen conocimientos que aumenten la comprensión de realidades y que proporcionan una guía hacia la acción.

Weaver y Oppermann (2000), incluyen como fuentes secundarias las recopilaciones 
estadísticas proporcionadas por investigaciones previas; en este sentido nos hemos basado en los datos cuantitativos de la tesis doctoral "el mundo sobre las olas. Perspectivas de desarrollo turístico a través del surfing" y en algunos artículos tanto de prensa generalista como de prensa especializada.

Una de las principales herramientas que se ha empleado para la obtención de datos primarios es la observación participante. El investigador ha podido interactuar durante la investigación de su tesis doctoral con los miembros que constituyen el objeto de estudio del presente artículo utilizando al menos tres de los cuatro roles que establece Junker (1960) y que recoge Valles (1999).

Téllez Infantes (2007) considera que cuando el investigador realiza trabajo de campo y ésta incluye una observación participante correcta, es necesaria una transformación del investigador, que algunos han denominado socialización. No se trata de un proceso de doble socialización cualquiera, es un proceso complejo pues nos encontramos con un adulto socializado previamente en otra cultura, por tanto, es posible que fuese más adecuado afirmar que se trata de un proceso de socialización secundaria o resocialización (Berger y Luckmann, 1984: 164). En este sentido, también existe una parte importante de socialización por parte del investigador social que aprenderá e interiorizará nuevos elementos de este nuevo medio ambiente y los integrará a la estructura de su personalidad, bajo la influencia de experiencias y de esta manera se adaptará al entorno social que debe vivir.

Para conseguir la mejor información posible para contrastar lo observado, primeramente se trató de identificar todos aquellos agentes implicados en la regulación del surf en la zona de playas, estableciendo la siguiente clasificación:

Organismos de gestión y ordenación de playas: Concejalías municipales, Diputación provincial, Patronato de turismo, Protección Civil, Capitanía Marítima, Subdelegación del gobierno, Salvamento y socorrismo, Guardia Civil, Federaciones de surf.

Prestadores de actividades de surf: Escuelas de surf, clubs deportivos

Usuarios de la playa: Surfista libre, bañistas, practicantes de otras actividades.

Las conversaciones son herramientas que se desprenden directamente de la observación participante, y se consideran una excelente fuente de datos primarios y un recurso técnico. Bajo la expresión "técnicas de conversación" se organizan varias técnicas cualitativas como las entrevistas en profundidad, biográficas y en grupo. (Valles, 1999: 176)

Para poder estar en facultad de realizar conversaciones dentro de nuestro campo de estudio, el investigador considera que se ha de tener un conocimiento bastante amplio del objeto de estudio al que nos enfrentamos, ya que el vocabulario específico de la subcultura surf es tremendamente amplio y complejo.

Por este motivo el investigador considera su socialización previa lo capacita y le facilita la elección de informantes para poder realizar estudios sobre el surf. Sin duda, debido a la gran complejidad del tema abordado la correcta elección de los informadores puede ser capital para validar o descartar nuestras hipótesis.

Las entrevistas de la investigación social se enmarcan en el conjunto de entrevistas profesionales. Según Valles (1999: 183) la expresión "entrevista en profundidad" (Gorden, 1956; Banaka, 1971) parece ser la más utilizada como concepto terminológico empleado, sin embargo, este autor considera hacer referencia a un glosario de conceptos que se incluyen dentro de esta expresión. Enumera pues, entrevista focalizada, entrevista estandarizada no programada, entrevista no estandarizada, entrevista especializada a élites y entrevista biográfica. (Valles, 1999: 183-184).

La naturaleza de este proyecto nos llevó a realizar entrevistas especializadas a élites, con unos objetivos muy marcados, y se considera que se obtuvo una "información difícil de obtener 
sin la mediación del entrevistador" (Valles, 1999: 197).

El investigador ha planteado este tipo de entrevistas con el objetivo de profundizar en algunos aspectos clave dentro del objeto de estudio. Cuando así ha sido posible, el investigador ha preferido realizar entrevistas presenciales, no estandarizadas. Pese a ello el investigador prepara un guión para cada entrevistado, el cual se revisa una vez que se ha finalizado la entrevista, antes de que el entrevistado se vaya, por si fuese necesario rescatar algún punto.

Como nuestra intención no es un análisis de legislación, aunque sea necesario comprenderla, sino un análisis de la realidad social a través de la percepción de lo que realmente sucede en los arenales, tras una profunda reflexión se decidió obtener información únicamente de aquellos con conocimiento pragmático de la situación. Realizando las siguientes entrevistas a élites:

Salvamento y socorrismo.

Entrevista a David Vallejo, socorrista en el arenal de A Lanzada en zona de práctica de surf y surfista con experiencia.

Escuelas de surf.

Entrevista a Yago Chamorro. Director de escuela con autorización para impartir clases en playa cercana A Lanzada.

Además de estas entrevistas, se trató de realizar un grupo de discusión con diferentes surfistas de la zona. Tanto las fechas como la complejidad de la temática hizo descartar esta opción. Posteriormente se planteó la posibilidad de distribuir el mismo cuestionario que se había distribuido en Santos (2015) con el que poder contrastar algunas de las respuestas obtenidas. Sin embargo, tras descartar dicha opción por las dificultades que entrañaban su distribución, aprovechando un artículo de un periódico local que destacaba el conflicto entre surfistas y bañistas en la playa de Patos (Nigrán) se realizó una pequeña consulta a través de la cuenta del blog Mareas Vivas en la red social Instagram y se planteó la pregunta “¿Consideras que hay que regular el surfing en verano?". Se obtuvieron 69 respuestas, de las cuales un 70\% (48) respondieron "si, al menos en la orilla" y un 30\% (21) respondieron que no.

Aunque la validez de esta encuesta es muy baja, y teniendo en cuenta que esta cuenta tiene seguidores a monitores y alumnos de escuelas, sirve para evidenciar que existe en el ambiente surfero la comprensión de una situación de conflicto entre los usuarios de las playas.

Las limitaciones de los datos cuantitativos disponibles han sido enormes en el desarrollo de esta investigación empírica. Se esperaba y se consideraba esencial la colaboración de la federación galega de surf para poder comprender la realidad cuantitativa en la playa que estábamos estudiando. Lamentablemente no se pudo tener acceso a los datos relativos al número de escuelas y número de cursillistas que desempeñan su actividad en los diferentes arenales gallegos. Datos que tenían especial interés para poder evidenciar la importancia de A Lanzada como destino de surf y a través de su análisis comparado conocer su evolución.

\section{HIPÓTESIS Y RESULTADOS}

Las hipótesis planteadas y que se pretenden validar o descartar son las siguientes:

1. Existe una necesidad de regular las zonas de práctica de surf en verano en la playa de $A$ Lanzada

Para tratar de refutar esta hipótesis, además de basarnos en la observación del investigador y posibles conclusiones que haya podido obtener, nos basaremos en las opiniones vertidas por 
las élites en las diferentes entrevistas realizadas.

Consideramos importante comenzar con la opinión de David Vallejo, quién en su entrevista a pie de playa, nos comenta que en la zona de la playa que está bajo su responsabilidad, el Ayuntamiento de Sanxenxo ha determinado establecer una zona de surf de 100 metros ampliable a 150 metros que coincide con la zona de entrada y salida de embarcaciones y con la zona de rompiente de olas más importante del arenal. Debemos recordar que el mayor volumen de playa pertenece al Ayuntamiento de O Grove. A este respecto David nos comenta que pese a que la concesión de la explotación de esta zona de surf (perteneciente geográficamente a Sanxenxo) es para la única escuela que licita este Ayuntamiento, en realidad la escuela concesionaria de O Grove, utiliza una zona vigilada por socorristas de Sanxenxo ya que dicho Ayuntamiento no establece regulación mediante banderines y es muy difícil establecer límites reales, por lo que en aras de la seguridad se toma esta decisión.

Con una longitud de dos mil quinientos metros de extensión total del arenal existen tres concesiones para explotar por escuelas de surf. Tartaruga Surf Center en el centro de la playa y en la izquierda Prado Surf Escola, ambas pertenecientes a O Grove, y un poquito más a la izquierda Mission Suf School, sita en terrenos de Sanxenxo.

Sobre la delimitación de la zona de surf, esta prohíbe expresamente el baño, y se colocan banderines para que los bañistas sepan que no pueden bañarse, pero muchos de ellos no hacen caso de estas limitaciones. En ocasiones han existido problemas ya que no asumen que en una zona de la playa en la que se bañaban regularmente, ahora no pueden hacerlo.

David como profesional y persona con experiencia en la playa como socorrista y surfista, considera que la limitación en dicha zona es correcta ya que se dan varios condicionantes que pueden hacer peligroso el baño, como son la presencia de rocas, de olas los días más grande y además así se reserva el acceso a embarcaciones. Por lo tanto, de elegir una zona para surf, sería esta. "Yo creo que las limitaciones al bañista en el Ayuntamiento de Sanxenxo están bien, otra cuestión es la convivencia entre surfistas libres y las escuelas" afirma David.

La zona surf en A Lanzada (Ayuntamiento de Sanxenxo) es realmente pequeña para las dimensiones de la playa, ya que sólo una pequeña parte del mismo pertenece a este municipio. Coincidiendo en esta pequeña parte del arenal, la escuela adjudicataria de la concesión, surfistas libres y accesos directos del parking al arenal, lo que supone que sea una de las zonas más concurridas. Además, se suma que debido a la mayor presencia de olas, la otra escuela concesionaria de esta parte de la playa, aun siendo de O Grove intenta pegarse lo más posible a la zona de Sanxenxo.

No es difícil imaginarse que algunos días de verano que puedan coincidir olas y buen tiempo, este fragmento de playa puede ser fuente de muchos conflictos. Por tanto si parece importante delimitar zonas de surf.

2. La regulación del surf es mejorable en A Lanzada.

Desde el punto de vista del investigador, regular el surf no es exclusivamente delimitar una zona de surf entre Junio y Septiembre, establecer unos pliegos con los que licitar la explotación exclusiva a través de concesiones durante la temporada de verano y pensar que así se ha solucionado algo.

La regulación debe responder a factores tan básicos como la sostenibilidad económica y social de todos los agentes implicados en el disfrute, explotación y posible explotación de este arenal.

Para buscar una sostenibilidad económica, y no perjudicar a otras escuelas que utilizan A Lanzada, parece un agravio no poder utilizar la zona de surf para realizar sus clases al igual que durante el invierno. De esta manera parece evidente que se limita la actividad de algunas escuelas que no consiguen concesión. Evidentemente aquella que quiera proceder a la 
instalación de caseta durante el verano a pie de playa debe participar en una licitación.

Mediante la aplicación de la técnica de observación de nuestro trabajo de campo, pudimos comprobar, gracias a las indicaciones a través de cartelería y banderolas, que en otras playas de la provincia (Patos, Nigrán), diferentes escuelas comparten zona de surf, garantizando así, no sólo que pueden practicar en verano en la misma playa que lo hacen durante el invierno, así como la libre elección de los alumnos.

A este respecto, Yago Chamorro, director de Riders73 escuela concesionaria de explotación en la playa de Foxos (Sanxenxo) nos explicó que en realidad algunas veces pueden impartir clases a playas que no son concesionarias en virtud de una serie de acuerdos firmados por la Asociación de Escuelas de la zona.

Parece que podría ser más sensato limitar el número de alumnos que cada escuela puede ofertar simultáneamente durante el verano, tratando de realizar un ajuste de volumen en relación a su actividad durante el invierno, por ejemplo, que limitar la presencia de escuelas no concesionarias.

Actualmente el único límite de volumen de alumnos es el que la escuela sea capaz de ofertar según su número de tablas y monitores. Circunstancia que tampoco parece muy lógica para cualquier persona que pueda reflexionar sobre la calidad del servicio que se puede ofrecer en una zona acotada si se masifica.

Por otra parte, David Vallejo, asegura que las escuelas de surf tampoco tienen horario de explotación, por lo que pueden realizar clases incluso antes de la llegada de los socorristas. Circunstancia que ve con buenos ojos y no le preocupa demasiado, ya que considera que los propios monitores hacen de "socorristas" con sus alumnos. "Si tienen alumnos a cualquier hora entran al agua", asegura.

Sobre mi propuesta de realizar una limitación de horarios, su gesto es de resignación. "No creo que cambie nada". Mi planteamiento tiene como base que por el pequeño tamaño de las olas, estas rompen en la orilla. Por las características de la playa, especialmente en marea alta, existe una zona de conflicto real entre surfistas y demás usuarios de la playa que no pueden disfrutar de la orilla.

Circunstancia que no sucede en otras playas como la vecina Foxos en la que las olas rompen alejadas de la orilla por lo que el riesgo de conflicto es menor.

Razón por la que, en virtud de la sostenibilidad social de surfistas y demás usuarios, desde el punto de vista del investigador, debería proponerse una limitación horaria "ad-hoc" para la playa pues no es lo mismo marea alta en mareas vivas a las 16 horas de un día de calor en un fin de semana del mes de Agosto, que un lunes lluvioso en cualquier condición en la que no existe riesgo de conflictos.

El investigador considera que la calidad turística no consiste en limitar, sino en regular de una manera que la satisfacción de todos los agentes de la playa sea lo más elevada posible.

La percepción asociada a la calidad está vinculada muchas veces a la forma en la que se comunica determinado hecho. Es decir, una correcta comunicación puede evitar malos entendidos. Por tanto parece importante comunicar de manera correcta cualquier circunstancia que pueda afectar al uso habitual de la playa por parte de los usuarios.

Además, las banderas que delimitan la zona de surf y prohíben el baño no son las únicas instaladas en la playa de A Lanzada, ambas escuelas ponen banderines publicitarios de promoción en la arena y aunque David Vallejo nos recalca que es una práctica prohibida, no se meten con ello.

En opinión del investigador, utilizar el mismo efecto visual puede producir que el bañista no lea los banderines, o piense que es un banderín con información de la escuela, no de los socorristas. 
Volviendo al ejemplo de la playa de Patos, no se han observado otras banderolas similares y además se han encontrado varios carteles explicativos sobre las limitaciones zonales de la playa.

Imagen 5. Carteles explicativos Surf Playa de Patos.

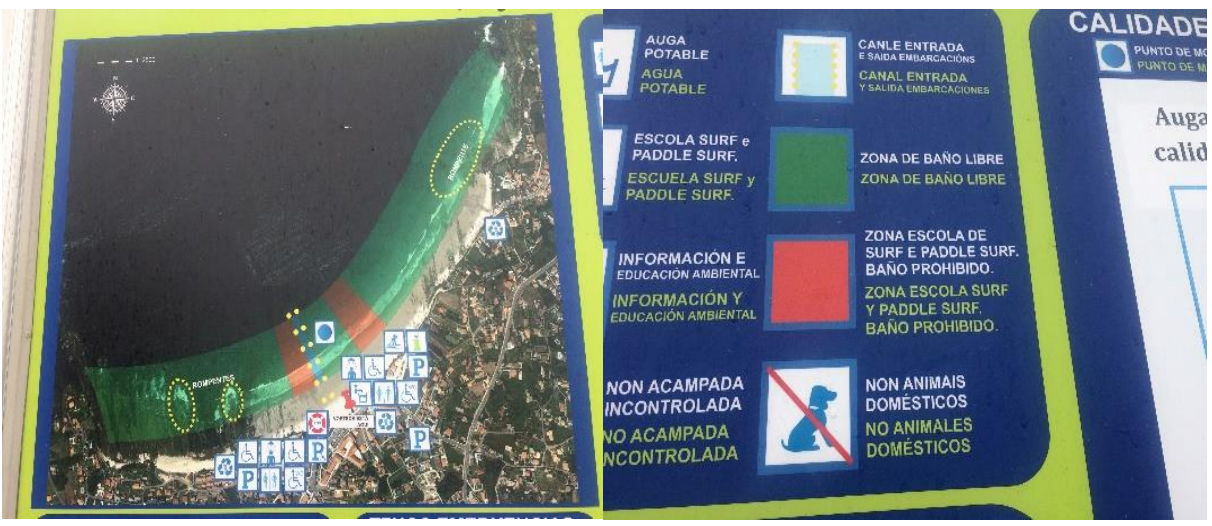

En algunas playas asturianas existe un servicio de megafonía que puede ser realmente útil para recordar la existencia de zonas prohibidas de baño. Quizás utilizar boyas de diferentes colores y otro tipo de banderolas podría facilitar el uso correcto de las delimitaciones de la playa.

Por tanto la conclusión es que la hipótesis es cierta y sería necesaria una revisión y una mejor comunicación de la regulación de surf que se realiza en la playa de A Lanzada.

3. El turismo de surf está descendiendo en A Lanzada durante el verano.

La ausencia de olas durante el verano hace que prácticamente la totalidad de turistas que practican surf a los que afecta es a aquellos que ven el surf como una actividad turística, ya que aquellos que el surf es su motivación principal del viaje elegirán otros destinos.

La observación por parte del investigador es que cada vez hay menos grupos de iniciación y que estos son menos numerosos de lo que eran hace algunos años. A este respecto, los socorristas nos informan que en general hay un menor número de turistas en las playas en los últimos años. Para hacer esta aseveración se basan fundamentalmente en el número de incidencias a las que han tenido que responder. Matizan además el fuerte descenso de turista internacional. "Antes venían al puesto turistas de lugares muy variopintos, ahora son casi todos españoles”. Parece evidente que si desciende el número de turistas, descenderá el número de demandantes de cursos de surf a pie de playa. Sobre esta circunstancia Yago Chamorro asegura que aunque el dispone de concesión y da servicio en Foxos, hace años que luchar por dar clases a los turistas. Había una guerra de precios brutal con el boom de las escuelas. Yo a día de hoy no pongo ni publicidad en los hoteles, me dedico a los veraneantes que repiten año tras año porque tienen aquí una casa o el típico turista que viene siempre, y entre ellos y el boca a boca cubro el verano. Una filosofía que sigo también en invierno que no busco colegios o grupos enormes, me dedico a aquellas personas que quieren mejorar conmigo, unos vienen todo el invierno y tienen un nivel medio y los que vienen en verano un nivel más bajo, pero raro es el grupo que viene a unas clases y no vuelve. 


\section{CONCLUSIONES}

Hemos podido comprender en este artículo que el turismo de surf es un concepto que engloba dos realidades muy diferentes. Por un lado podemos referirnos a aquellas personas cuya motivación principal para realizar un viaje es la de deslizarse sobre las olas, pero también incluye a todos aquellos turistas que ven en el surf una actividad de ocio para realizar en sus vacaciones. Además, hemos podido comprobar que gracias a la imagen de libertad y fotogeneidad de las tablas, olas y playas, es un atractivo turístico que encaja perfectamente en la sociedad actual.

Se ha tratado de argumentar las diferentes razones por las que los destinos deben conocer la tipología de turismo de surf que reciben y en base a ello tratar de ofrecer una regulación lo más satisfactoria posible para todos los agentes que conviven en la playa.

Hemos tratado de reflexionar sobre la normativa desde una perspectiva social y no legal, exponiendo aspectos positivos, negativos y posibilidades de mejora para las delimitaciones de las zonas de surf en el estudio de caso de A Lanzada y hemos destacado la importancia de la búsqueda de una sostenibilidad económica para la industria de surf local que permita concesiones, pero no a costa de expulsar a otras escuelas que utilizan esa playa fuera de la temporada de verano. Subrayamos también la necesidad de encontrar un punto de equilibrio en la normativa que garantice una sostenibilidad social para que los turistas tradicionales, usuarios de A Lanzada no se vean desplazados por los surfistas, ya no sólo por respeto, sino por no generar rechazo del turista tradicional al premiar al turista que ve en el surf a un producto turístico en la actualidad.

Se ha evidenciado como los patronatos de turismo prestan más atención a la promoción que a la planificación o regulación en la Playa de A Lanzada, realidad que contrasta con lo que sucede en otros arenales como Somo o Salinas. Pese a ello, parece que la situación actual del surf en la playa ha mejorado gracias a las concesiones ya que la autoregulación producía numerosos incidentes.

Parece evidente que hay bastante margen de mejora en la gestión de las zonas de surf, especialmente en el uso de estas zonas por parte de escuelas, demostrándose necesarios incorporar ciertos límites en cuanto al volumen y horario de cursillos de surf, además de posibles inspecciones que verifiquen el cumplimiento de la diferente normativa legal y tributaria.

Existe un desconocimiento por parte de los bañistas de sus obligaciones como usuarios de la playa. A este desconocimiento se suma una comunicación deficiente de los espacios de baño.

Además, se ha demostrado que la regulación de playas para la práctica de surf es lo suficientemente compleja para que no haya un criterio general como sucede con los chiringuitos. Parece lógico desarrollar una regulación «ad hoc» considerando las singularidades de cada playa y de cada día de verano.

Verdaderamente es un tema complicado, pero creo que con una estrecha colaboración entre planificadores de territorio locales, Administración del Estado, dueños de las escuelas y usuarios de las playas podríamos llegar a una situación satisfactoria para todos.

\section{BIBLIOGRAFÍA}

Álvarez Sousa, A. (2011) . Sociología del turismo. Madrid: Udima

Barilotti, S. (2002) . Lost Horizons: Surfer colonialism in the 21st century. The Surfer's Path, vol. Oct/Nov, pp. 30-39. 
Berger, P.L. y Luckman, T. (1967) . La construcción social de la realidad. 2008. Buenos Aires: Amorrortu.

Buckley, R. (1999) . Recreational Capacity for Surf Tourism in Indonesia's Mentawai Islands. International Symposium on Society and Resource Management. S.l.: s.n.,

Esparza, D. (2014). La historia del surf en España. Alemania: Amazon. George, S. (2007) "Pre-contact: the surfing tradition of Sao Tome". Surfers Journal, 16 (3): 40-49.

Gilmore, Heath (15 July 2007)."Bra Boys say it's Ours and we'll fight for it".The Sydney Morning Herald.

Henderson, M. (1999). "Some Tales of Two Mags: Sports magazines as glossy reserviours of male fantasy". Journal of Australian Studies, September, pp. 64-80.

Henderson, M. (2001). "A Shifting Line Up: Men, women and Tracks surfing magazine". Journal of Media and Cultural Studies, 15(3): 319-332.

Hibbard, D. y Salbosa, A. (2006). Designing Paradise. USA: Princeton Architectural Press.

Junker, B. (1960). Field work: An introduction to the social sciences. USA: University of Chicago Press.

Kampion, D. (2003). Stoked! A history of surf culture. USA: Gibbs Smith.

Nazer, D. (2004). “The tragicomedy of surfers commons". Deakin Law Review, 9 (2): 665-713

Santos, D. (2015). Desarrollo turístico a través del surf. Tesis Doctoral. Madrid: Universidad Rey Juan Carlos

Santos González, D. (2017). "El surf, de la práctica en la naturaleza a deporte de piscinas", en Díaz Cano, E. y Barbeito Iglesias, R. XV Premio de Ensayo Breve en Ciencias Sociales "Fermín Caballero". Toledo: ACMS, pp.87-107

Téllez Infantes, A. (2007). La investigación antropológica. España: Editorial Club Universitaria..

Valles, M. (1999). Técnicas cualitativas de investigación social. Reflexión metodológica y práctica profesional. España: Síntesis.

Wayne Hull, S. y Wayne, S. (1976). A sociological study of the surfing subculture in the Santa Cruz area A. S.l.: San Jose State University.

\section{Breve currículo:}

\section{Diego Santos González}

Diego Santos González (Pontevedra, 1984) doctor en Sociología (Universidad Rey Juan Carlos, 2016) ha sido capaz de conjugar sus inquietudes intelectuales, sus pasiones personales y profesionales en una tesis doctoral titulada "El mundo sobre las olas. Perspectiva de desarrollo turístico a través del surfing". Nacer en una familia de agricultores, artistas y deportistas, marcan su personalidad y sus inquietudes personales, por lo que el surf, los viajes, la escritura y la vida rural, son siempre el eje de sus relatos e investigaciones sociales.

\section{Jose Miguel Moreno Carrillo}

Licenciado en Sociología y Diplomado en Estudios Avanzados (DEA), por la Universidad Pontificia de Salamanca (UPSAM), actualmente concluye su tesis doctoral en la Universidad de Castilla La Mancha. Licenciado en Ciencias Políticas y de la Admón., Universidad San Pablo CEU de Madrid; Experto Universitario en Análisis Psicosocial de la Violencia de Género, Universidad de las Islas Baleares (UIB); Experto Profesional en Dirección y Gestión de Seguridad Integral (UNED), con la habilitación de Director de Seguridad Privada (Dirección General de la Policía-Ministerio del Interior); Diplomado en Aptitud Pedagógica (CAP), especialidad de Geografía e Historia, Facultad de Ciencias de la Educación (UCM). 